\title{
Weak fault feature extraction of gear based on KVMD and singular value difference spectrum
}

\author{
Hongkun Li ${ }^{-}$, Chaoge Wang, Mengfan Hou, Rui Yang, and Daolong Tang
}

School of Mechanical Engineering, Dalian University of Technology, Dalian 116024, People 's Republic of China

\begin{abstract}
Gearbox is an important component of many industrial applications. When the gear fault occurs, the vibration signal is characterized by multi-component, multi-frequency modulation, low signal to noise ratio, weak fault characteristics and difficult to extract. This paper proposes a gear fault feature extraction method based on improved variational mode decomposition(VMD) and singular value difference spectrum. Firstly, the method is optimized for the decomposition level $\mathrm{K}$ of the VMD algorithm, and an improved method of VMD decomposition layer number $\mathrm{K}$ for central frequency screening (KVMD) is proposed. Then, the gear fault vibration signal is decomposed into a series of bandlimited intrinsic mode functions using KVMD. Due to the interference of the noise, it is difficult to make the correct judgment of fault in the spectrum of each mode component. According to the correlation coefficient criterion, the components with larger correlation coefficients are chosen to singular value decomposition. The singular value difference spectrum is obtained, and the effective order of the reconstructed signal is determined from the difference spectrum to denoise the signal; Finally, the processed signal is analyzed by Hilbert envelope. The fault characteristic frequency can be extracted accurately from the envelope spectrum. Through the analysis of the experimental data of gear fault, the results show that the method can effectively reduce the influence of the noise, and accurately realize the extraction of gear fault feature information.
\end{abstract}

\section{Introduction}

Gear box is an important transmission device in mechanical equipment, which plays a role of transmission speed and force, and its operation state directly affects the working accuracy and production efficiency of the whole equipment [1]. When the gear fault occurs in early stage, the acceleration signal obtained from the gearbox shell contains the vibration signals of many gears and the noise signals produced by the noise source, which belongs to the typical nonlinear and non-stationary signal [2]. The time-frequency methods of EMD and LMD are especially suitable for the analysis and processing of nonlinear non-stationary signals [3-5].

Corresponding author: lihk@dlut.edu.cn 
However, the EMD and LMD methods exist in the problem of modal aliasing. In view of the shortcomings of these methods, Dragomiretskiy [6] proposed variational mode decomposition (VMD) in 2014, which is a new adaptive signal analysis method. It can transform the signal decomposition process into the variational frame, and use the nonrecursive processing method to realize the division of the signal in the whole frequency domain and the effective separation of each component. Compared with EMD and LMD, VMD is equivalent to a group of adaptive Wiener filters, abandoning the constraint of recursive mode decomposition [7,8]. Therefore, the modal aliasing inherent in EMD and LMD methods can be effectively avoided.

In this paper, the VMD method is used to deal with the early fault signal of the gear box with low signal to noise. In order to further suppress the noise components in the obtained signal components and achieve the accurate extraction of the characteristic frequency, the singular value difference spectrum method is used as the post-processing of the VMD $[9,10]$. A method of gear weak fault feature extraction based on improved variational mode decomposition and singular value difference spectrum is proposed. The analysis results of the measured data of the gearbox verify the effectiveness of the method.

\section{Basic theory}

\subsection{The method of KVMD}

In VMD decomposition, users need to preset the number of decomposition layers. After the original signal is decomposed by $\mathrm{VMD}$, and $K$ intrinsic mode functions are obtained [6], and each IMF component has a frequency center $\omega_{k}(t)$. There is a close relationship between the decomposition layer $K$ and $\omega_{k}(t)$, and whether the $K$ value is set properly or not will directly determine the decomposition effect [7,8]. The value of $K$ is too small, the signal will not be decomposed thoroughly, the value of $K$ is too large, the signal will appear over decomposition phenomenon. In this paper, the decomposition layer $K$ value of the VMD algorithm is optimized, and the improved method of the variable mode decomposition layer number for the center frequency screening is proposed. The principle is that the $K$ value is determined by comparing the ratio of the adjacent two central frequencies to the threshold value at the same $K$ value. After a large number of experiments, the threshold value is 1.1 .

In summary, the specific steps of the KVMD algorithm are as follows:

Step 1: Initialize the $K$ value.

Step 2: Use the VMD algorithm to decompose the signal, obtain the $K$ intrinsic mode functions, and the central frequency of each component $\omega_{k}(t)$.

Step 3: Let the center frequency of the latter component $\mathrm{IMF}_{K}$, except the center frequency of the previous component $\mathrm{IMF}_{K-1}$, obtain $K-1$ frequency ratios $\lambda_{1}, \lambda_{2}, \cdots, \lambda_{K-1}\left(\lambda_{K}=\omega_{K-1} / \omega_{K}\right)$.

Step 4: Set threshold $\theta$, when $\lambda_{K}>\theta$, think VMD decomposition is not complete enough, make $K=k+1$, return step (2) (3).

Step 5: When $\lambda_{K} \leq \theta$, the component of $\mathrm{IMF}_{K}$ and $\mathrm{IMF}_{K-1}$ can determine the frequency aliasing, and the VMD appears to be over decomposed. Therefore, the results $K=K-1$ are obtained and the decomposition result is output.

\subsection{Singular value difference spectrum}


Setting $Y=(y(1), y(2), \ldots, y(N))$ is the actual fault vibration signal, which is a set of discrete digital signals, and the $m \times n$ order Hankel matrix constructed by this signal is as follows:

$$
H=\left[\begin{array}{cccc}
y(1) & y(2) & \cdots & y(n) \\
y(2) & y(3) & \cdots & y(n+1) \\
\vdots & \vdots & \vdots & \vdots \\
y(N-n+1) & y(N-n+2) & \cdots & y(N)
\end{array}\right]
$$

where $N$ is the length of the signal, $1<n<N$. Order $m=N-n+1$, then $H \in R^{m \times n}$.

The singular value decomposition of matrix $H$ can be obtained:

$$
H=U S V^{T}
$$

where $U=\left(u_{1}, u_{2}, \ldots, u_{m}\right) \in R^{m \times n}$ and $V=\left(v_{1}, v_{2}, \ldots, v_{n}\right) \in R^{m \times n}$ are a set of orthogonal matrices $S=\left(\operatorname{diag}\left(\delta_{1}, \delta_{2}, \ldots, \delta_{q}\right), 0\right)$.or its transpose is determined by $m \leq n$ or $m>n$,stands for zero matrix, $D \in R^{m \times n}, q=\min (m, n)$, and $\delta_{1} \geq \delta_{2} \geq \ldots \geq \delta_{q} \geq 0$ is called the singular value of matrix $H$ [9].

Let the sequence of all singular values in descending order be: $\delta_{i}(i=1,2, \ldots, q)$, then:

$$
b_{i}=\delta_{i}-\delta_{i+1}, i=1,2, \ldots, q-1
$$

The sequence composed of all $b_{i}$ is called singular value difference spectrum. By the definition of the difference spectrum [10], the peak in the difference spectrum is caused by the correlation between the noise and the useful signal. If the maximum mutation position occurs at point $s$, the order prior to the $s$ point can be reconstructed to reduce noise.

\section{Experimental analysis}

In order to verify the reliability and validity of the method, the gear root crack fault experiment is simulated on the comprehensive test-bed of power transmission fault diagnosis, as shown in Figure 1.

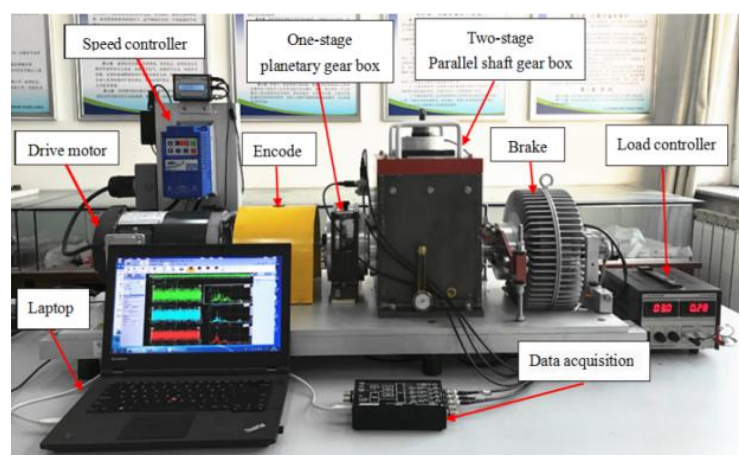

(a) Gear box test bench

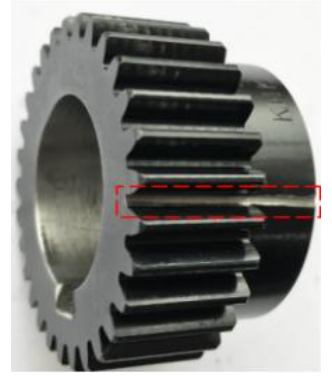

(b) Fault gear

Figure 1. Comprehensive test bench for power transmission fault diagnosis. (a)Gear box test bench; (b) Fault gear. 
The experimental platform is mainly composed of variable speed drive motor, coupling, torsion sensor and encoder, one-stage reducer planetary gear box (Deceleration ratio is 4.572:1), two-stage increase parallel shaft gear box, programmable magnetic brake and so on. In the experiment, the acceleration sensors are installed on the vertical, horizontal, radial and axial test points of the right end cover of the intermediate shaft of the parallel shaft gear box.

The parallel shaft gear box is composed of two pairs of gear pairs. The internal structure modeling of gearbox is shown in Figure 2. The modulus of gear is 1.5, the material is $\mathrm{S} 45 \mathrm{C}$, the number of teeth is $Z_{1}=100, Z_{2}=29, Z_{3}=90, Z_{4}=36$. By means of wire cutting, small cracks with width of $0.15 \mathrm{~mm}$ and depth of $1 \mathrm{~mm}$ were produced in the tooth root direction of gear $Z_{2}$ as early failures. In the experiment, the sampling frequency is $5120 \mathrm{~Hz}$, the length of the data is 4096 points. The motor speed is $1500 \mathrm{r} / \mathrm{min}$ (corresponding rotating frequency is $25 \mathrm{~Hz}$, and the gear fault characteristic frequency is $18.8 \mathrm{~Hz}$ ).

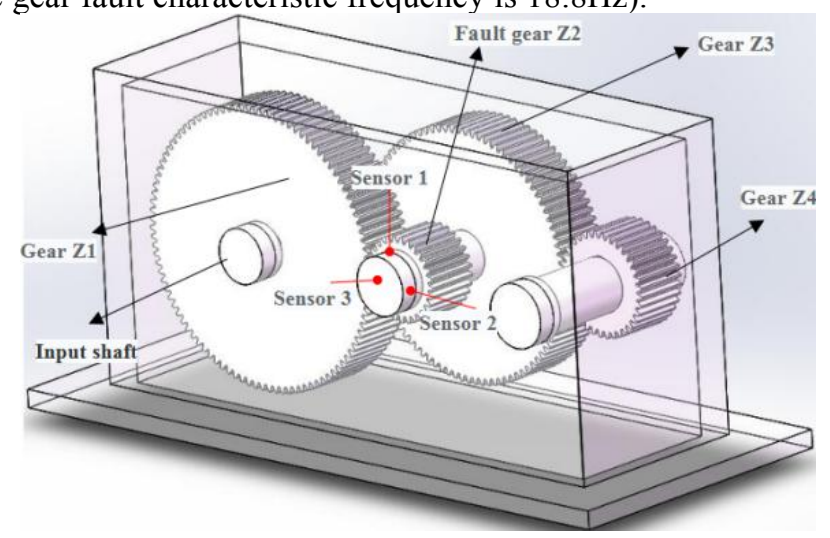

Figure 2. Internal structure model of parallel shaft gear box and position of sensor point.

The time domain waveform and spectrum of the tooth root crack fault are shown in Figure 3. In order to extract the fault characteristic information, the gear fault signals are decomposed by KVMD, and each modal component and its spectrum are obtained, shown as Figure 4. Then, according to the criterion of correlation coefficient, the U2 component with larger correlation number is chosen as the research object, and the Hankel matrix is constructed and singular value decomposition is carried out. In order to show the change of singular value clearly and intuitively, the first 50 data points of singular value and difference spectrum are plotted in the same coordinate system, as shown in Figure 5.
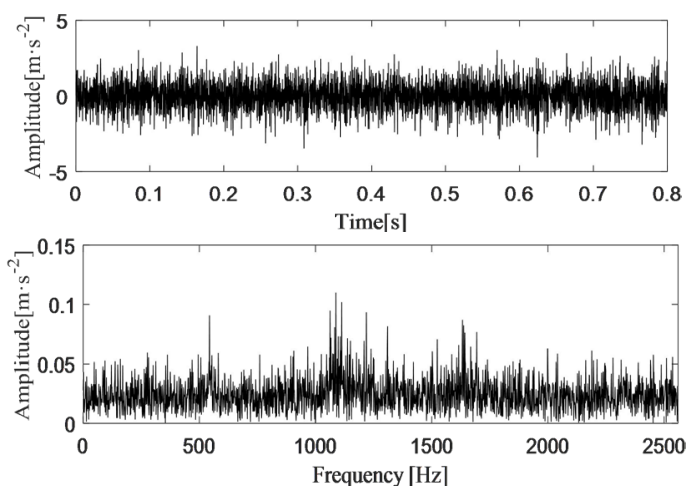

Figure 3. Waveform and Spectrum of Gear Fault Signal. 

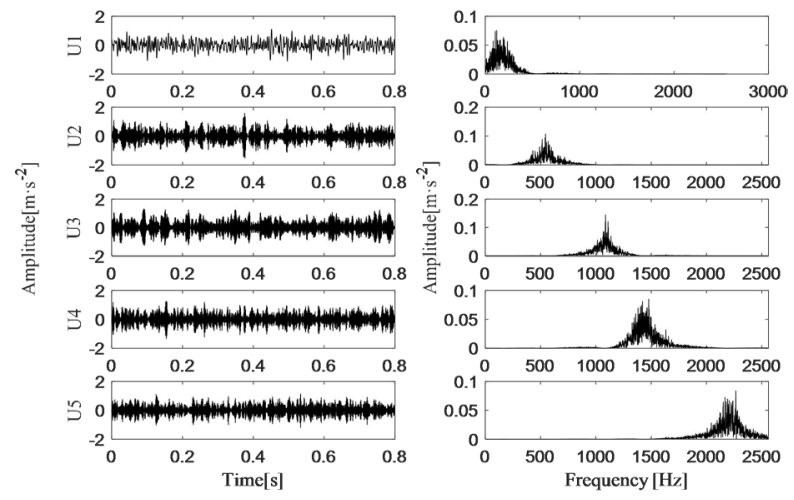

Figure 4. Modal Components and Spectrum of KVMD Decomposition.

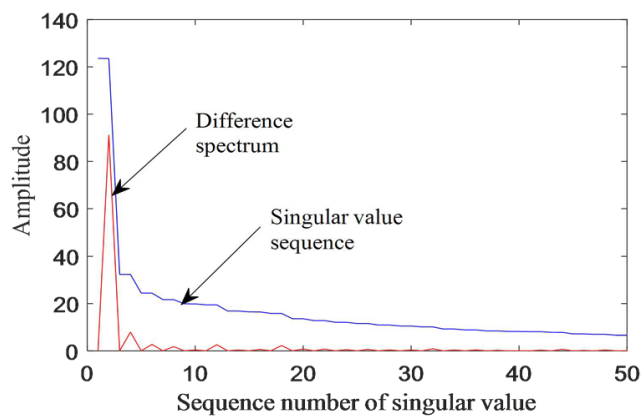

Figure 5. First 50 Points of Singular Value and Difference Spectrum.

Figure 5 shows that the coordinate position of the largest mutation in the differential spectrum occurs at fourth points, so the first 4 singular values are selected to reconstruct the singular values, and the reconstructed signal shown as Figure 6(a) is obtained. It can be seen from the Figure 6(a) that the equal time interval impact is approximately $0.53 \mathrm{~s}$ in the time domain waveform of the reconstructed signal, the reciprocal is $18.8 \mathrm{~Hz}$. Finally, the reconstructed signal is processed by Hilbert envelope. In the envelope spectrum shown in Figure $6(\mathrm{~b})$, an obvious peak value is observed at $18.8 \mathrm{~Hz}$, which corresponds to the fault characteristic frequency of the intermediate shaft pinion. So far, the method has successfully realized the early weak fault feature extraction of gear.

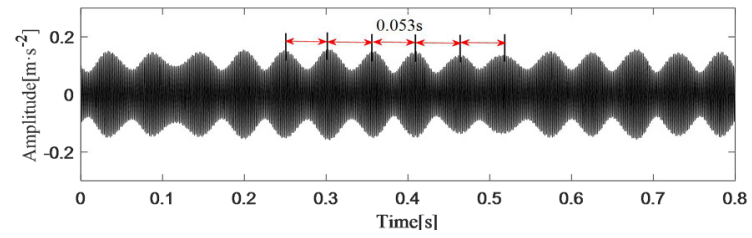

(a) Time domain waveform of reconstructed signal

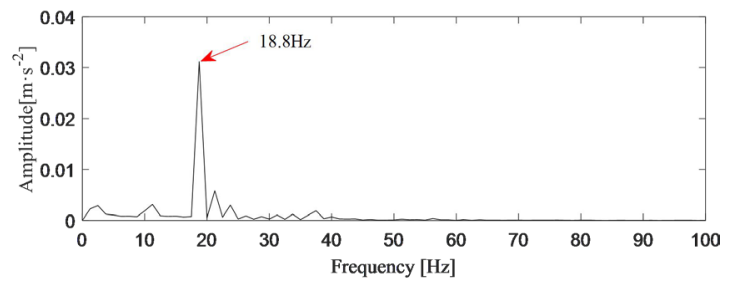

(b) Envelope spectrum of reconstructed signals 
Figure 6. The processing result of gear fault signal. (a) Time domain waveform of reconstructed signal; (b) Envelope spectrum of reconstructed signals.

As a comparison, the U2 component is directly processed by Hilbert envelope, and the results are shown in Figure 7. Although you can see there are peaks distributed in $18.8 \mathrm{~Hz}$, but there are many other interference frequency components, is not conducive to gear fault diagnosis, so the effect was not as good as in Figure 6(b) clear.

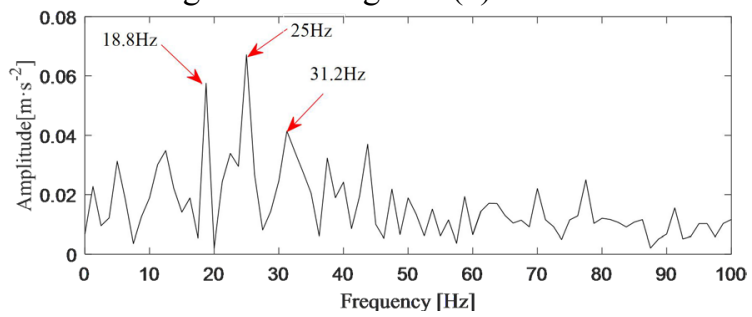

Figure 7. Envelope Spectrum of U2 Component.

\section{Conclusions}

In this paper, a fault diagnosis method based on KVMD and singular value difference spectrum is studied. Through gear fault test, it is proved that the method has strong recognition ability to the gear weak fault feature information in the strong noise background. KVMD method is used to optimize the number of decomposition layers in VMD algorithm, which enhances the adaptability of VMD decomposition. The fault signals of the gearbox are decomposed by KVMD, and the complex multi-component signals can be adaptively decomposed into a series of single component intrinsic mode components. The results of the decomposition effectively avoid the modal aliasing. In the singular value difference spectrum, the effective recombination order can be selected automatically and accurately, and the signal reconstruction can be realized, so as to eliminate the noise interference and highlight the impact of the fault. The combination of KVMD and singular value difference spectrum can accurately extract the weak fault feature information of gear, which provides a feasible new method for practical engineering application.

The work was supported by the Natural Science Foundation of China under Grant No. 51575075 and Collaborative Innovation Center of Major Machine Manufacturing in Liaoning for this research is gratefully acknowledged.

\section{References}

[1] V. Sharma, A. Parey. Meas 90 (2016) 560-575.

[2] B.Li, X.N Zhang, J.L. Wu, Mech Syst Signal Pr 85 (2017) 415-428.

[3] Q. Gao, C. Duan, H. Fan, Q Meng, Mech Syst Signal Pr 22 (2008) 1072-1081.

[4] R. Ricci, P. Pennacchi, Mech Syst Signal Pr 25 (2011) 821-838.

[5] J.S. Cheng, Y. Yang, Y. Yang, Digital Signal Process 22 (2) (2012) 356-366.

[6] K.Dragomiretskiy, D.Zosso. IEEE Tran on Signal Process, 62(3)(2014) 531-544.

[7] Y.Y. Liu, G.L. Yang, M. Li. Signal Process 125 (2016) 349-364.

[8] M. Zhang, Z.N.Jiang, K.Feng, Mech Syst Signal Pr 93 (2017) 460-493.

[9] M. Zhao, X.D Jia, Mech Syst Signal Pr 94 (2017) 129-147.

[10] X.Z. Zhao, B.Y. Ye, Mech Syst Signal Pr 25 (2011) 1617-1631. 